\title{
Garrapatas (Acari: Ixodidae) prevalentes en caninos no migrantes del noroccidente de Bogotá, Colombia
}

\author{
Edwar Javier Acero M.1-2, Omar Javier Calixto', Andrés Camilo Prieto' \\ 1. Facultad de Medicina Universidad Militar Nueva Granada- Grupo Patogenicidad Microbiana. \\ 2. Facultad de Medicina Universidad de la Sabana Grupo PROSEIM.
}

Correspondencia: edward.acero@unisabana.edu.co

Recibido: 24/06/2011 - Aceptado: 14/10/2011

\begin{abstract}
Resumen
El presente trabajo tuvo como objetivo identificar garrapatas duras (Acari: Ixodidae) en caninos domiciliados dentro de la ciudad de Bogotá, el riesgo poblacional de zoonosis trasmitidas por las garrapatas identificadas Rhipicephalus sanguineus, Rhipicephalus (Boophilus) microplus y Amblyomma maculatum, generan la necesidad de cambios de los paradigmas que orientan las políticas en salud pública. Para el desarrollo del estudio se trabajo en tres clínicas veterinarias particulares durante el año 2010. Se recolectaron garrapatas de caninos domiciliados y que demostraron sus propietarios estancia permanente dentro de la ciudad de Bogotá. Las garrapatas se colectaron de forma manual y se fijaron en Formol al $10 \%$. Un total de 799 canidos cumplieron el criterio de inclusión, se hallaron un total de 34 garrapatas (una por animal) lo que constituyó una tasa de infestación de 4,25\%.

Palabras clave: Rhipicephalus sanguineus, Rhipicephalus (Boophilus) microplus, Amblyoma maculantum, Calentamiento Global

Abstract

Ticks (Acari: Ixodidae) prevalent in canine non-migrants in northwestern Bogotá, Colombia

This is the first study that identifies hard ticks (Acari: Ixodidae) in dogs residing in the city of Bogota, changes in the paradigms that guide public health policies are becoming necessary the population risk of zoonosis transmitted Rhipicephalus sanguineus by ticks, Rhipicephalus (Boophilus) microplus and Amblyomma maculatum. Three private veterinary clinics during 2010 were collected ticks and domiciled canines showed their own permanent residence within the city of Bogota. Ticks were collected manually and fixed in formol 10\%. A total of 799 dogs met the inclusion criteria, there were a total of 34 ticks (one per animal), which was an infestation rate of $4.25 \%$.

Key words: Rhipicephalus sanguineus, Rhipicephalus (Boophilus) microplus, Amblyoma maculantum, Global warming
\end{abstract}

\section{Introducción}

La población humana está ocasionando modificaciones sin precedentes en el medio ambiente (1). La constante búsqueda de bienestar y desarrollo económico con uso indiscriminado de combustibles fósiles provoca cambios climáticos que afectan sistemas físicos y biológicos en todo el mundo $(2,3)$. En los últimos 100 años la temperatura mundial ha aumentado aproximadamente $0.75^{\circ} \mathrm{C}$ y desde hace 25 
años lo hace a una tasa $0.13^{\circ} \mathrm{C}$ por década $(4,7)$. En la ciudad de Bogotá Colombia latitud norte $4^{\circ} 035^{\prime}$, longitud $74^{\circ} 4^{\prime}$ y altura $2640 \mathrm{msnm}$ (8), los aumentos de temperatura desde los sesenta con significancia estadística en los 80 comienzos de los $90(9,10)$ y con aumentos históricos de temperaturas medias, máximas y mínimas en la primera década de siglo XXI, así como la reducción de las precipitaciones, reflejan el impacto del cambio climático (10). En salud se ha reconocido el calentamiento global como factor que predispone a la variación y aumento de la distribución geográfica de garrapatas y su picadura $(7,11-14)$, al establecerse en regiones históricamente libres de ellas (15-17) convirtiéndose en un problema adicional de salud publica asociado al cambio climático (18), por ser vector en comunidades humanas de zoonosis virales como fiebre hemorrágica del Congo (19) encefalitis (20), zoonosis bacterianas como borreliosis $(21,22)$, ehrlichiosis y anaplasmosis (22), y zoonosis parasitarias como babesiosis (23).

En Colombia están descritas garrapatas (Acari: Ixodidae) como Amblyoma cajennense, Rhipicephalus sanguineus, Boophilus microplus y Anocentor nitens $(24,25)$ y cerca a Bogotá se ha documentado presencia de Amblyomma cajennense, Rhipicephalus sanguineus, y Anocentor nitens con prevalencia de Ehrlichia spp y Rickettsia spp en caninos y equinos (26); en Tobia, Cundinamarca, entre 1934 y 1936, se realizó el primer reporte colombiano de una rickettsiosis humana de alta mortalidad transmitida por garrapatas, la cual se conoció como enfermedad de Tobia (27). Igualmente, durante el 2003 y el 2004 se realizó el segundo reporte colombiano fatal de rickettsiosis trasmitida por estos vectores en Tobia y Villeta, Cundinamarca (28). Este es el primer estudio que pretende identificar las garrapatas (Acari: Ixodidae) prevalentes en caninos no migrantes de el noroccidente de Bogotá - Colombia.

\section{Materiales y métodos}

Durante enero de 2010 a diciembre de 2010 se recolectaron garrapatas en 3 clínicas veterinarias particulares del noroccidente de Bogotá, llamadas respectivamente Clínica 1 con coordenadas Lat N. $4^{\circ} 43^{\prime} 47.16^{\prime \prime}$ y, $74^{\circ} 8^{\prime} 59.63^{\prime \prime} \mathrm{W}$, Clínica 2 con coordenadas Lat N. $4^{\circ} 41^{\prime} 40.98^{\prime \prime}$ y $74^{\circ} 5^{\prime} 38.97^{\prime \prime}$ W ubicadas en la localidad de Engativá y clínica 3 con coordenadas Lat N 4 $45^{\prime} 42.03^{\prime \prime}$ y $74^{\circ} 6^{\prime} 18.04^{\prime \prime} \mathrm{W}$ en la localidad de Suba. El principal criterio de inclusión para la colección de garrapatas en la consulta veterinaria fue la demostración por parte de los propietarios de los animales de su estancia permanente en la ciudad de Bogotá. Se hizo relación de animales totales Vs. aquellos que tenían garrapatas, calculando así la tasa de infestación. Las garrapatas se colectaron de forma manual y se fijaron en formol al 10\%.

En el laboratorio de Microbiología de la Facultad de Medicina de la Universidad Militar Nueva Granada se examinaron al estereomicroscopio marca Zeiss EF-75 y se identificaron usando claves taxonómicas pictóricas (24) y nomencladas (25, 29, 30). Se tomaron fotografías con cámara digital (Pentax Ra.45), y se midieron longitudes corporales totales así como estructuras del capítulo, hipostoma, palpos y sus segmentos con fines taxonómicos. Las fotos fueron manejadas en PC con Sofware Analizador de Imágenes licenciado Motic Versión 4.61. Se correlacionó con el mes de colección de garrapatas, la temperatura promedio, máxima, minina y humedad relativa promedio mensual, reportada en la estación meteorológica aeroportuaria de la ciudad, con coordenadas $4^{\circ} 42^{\prime} 5.74^{\prime \prime} \mathrm{N}, 74^{\circ} 8^{\prime} 49.01^{\prime \prime} \mathrm{W}(31)$.

\section{Resultados}

De un total de 799 canidos atendidos en consulta veterinaria general que cumplieron el criterio de inclusión Tabla 1, se hallaron un total de 34 garrapatas, una por animal, lo que constituyó una tasa de infestación de 4,25\%.

De las garrapatas recolectadas Tabla 1, (Acari: Ixodidae) se clasificaron 22 de la especie Rhipicephalus sanguineus, 11 de la especie Rhipicephalus (Boophilus) microplus y 1 de la especie Amblyoma maculantum. De $R$. sanguineus 14 fueron hembras y ocho machos; de Rhipicephalus (Boophilus) microplus 7 fueron hembras y 4 machos, de Amblyoma maculatum fue hallado sólo 1 macho, Tabla 2. Los especímenes fueron recolectados en todos los meses del año Tabla 3. La identificación de las especies $R$. sanguineus, Rhipicephalus (Boophilus) microplus y Amblyoma maculatum se confirmó basado en diferencias 


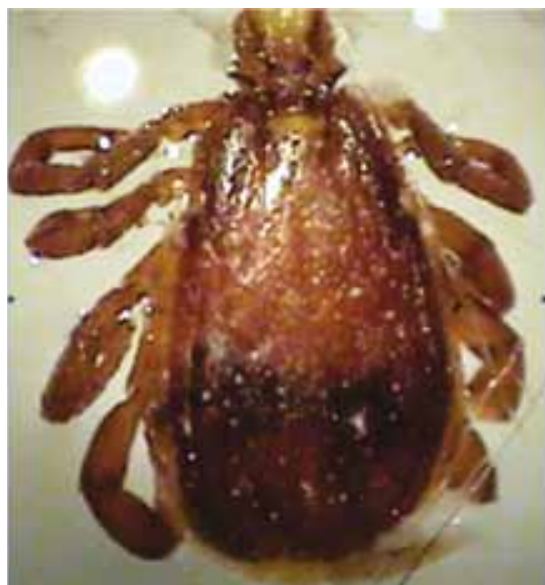

$1 \mathrm{a}$

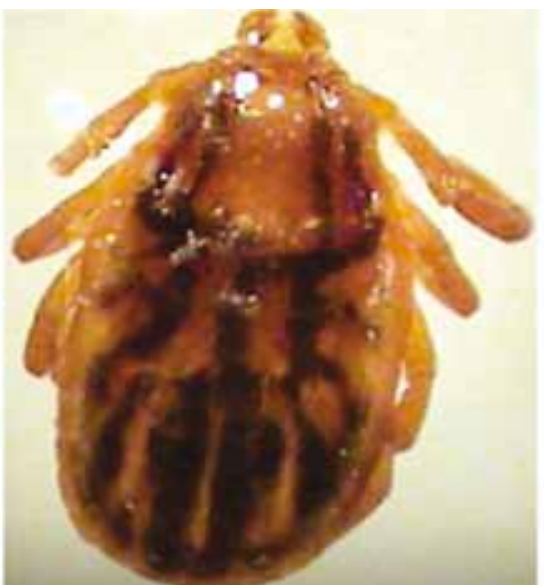

$1 \mathbf{b}$

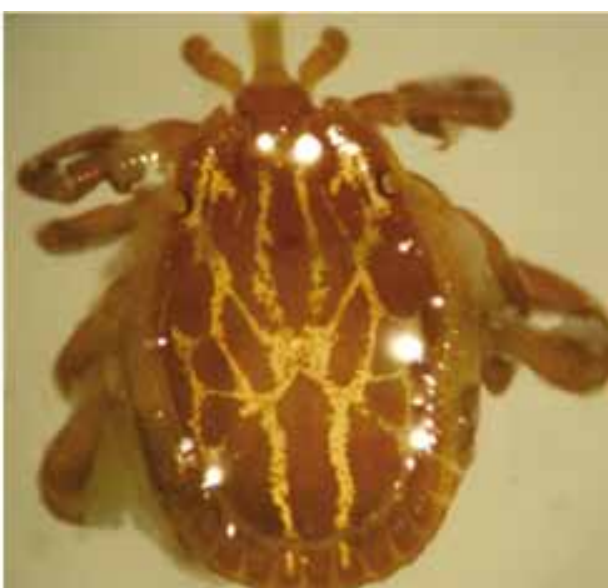

1c

Figura 1. Vista dorsal a $5 \mathrm{X}$, en especimenes machos de garrapatas Acari: Ixodidaes especies (a) R. sanguineus, (b) R. Boophilus microplus y (c) Amblyoma maculatum.

Tabla 1. Identificación y ubicación geográfica de las clínicas veterinarias con cantidad de garrapatas colectadas en la ciudad de Bogotá entre enero de 2010 a diciembre de 2010.

\begin{tabular}{cccc} 
Localidad & Clínica & $\begin{array}{c}\text { Coordenadas } \\
\text { geográficas }\end{array}$ & $\begin{array}{c}\text { Total cantidad de } \\
\text { garrapatas }\end{array}$ \\
Engativá & Uno - Osorio & Lat Norte. 4 43' $47.16^{\prime \prime}$ y & 25 \\
Engativá & Dos- Rincón & $74^{\circ} 8^{\prime} 59.63^{\prime \prime} \mathrm{W}$ & 5 \\
Suba & Lat Norte. & 9 \\
\hline
\end{tabular}

Tabla 2. Distribución por especie y género sexual de las garrapatas (Acari-Ixodidae) colectadas en Bogotá entre enero de 2010 a diciembre de 2010.

\section{Especie de garrapata}

Rhipicephalus sanguineus

Rhipicephalus (Boophilus) microplus

Amblyoma maculatum

\section{Hembras}

14

7

7

\section{Machos}

8

4

Cantidad total de garrapatas

Tabla 3. Número de garrapatas colectadas por mes y registro de temperaturas promedios mensuales, máximas, mininas y humedad relativa relacionadas entre enero de 2010 a diciembre de 2010 .

\begin{tabular}{|lccccc}
\hline \multicolumn{1}{c}{ Año $\mathbf{2 0 1 0}$} & $\begin{array}{c}\text { Número de } \\
\text { garrapatas } \\
\text { colectadas }\end{array}$ & $\begin{array}{c}\text { Promedio mes } \\
\text { temperatura en }{ }^{\circ} \mathbf{C}\end{array}$ & $\begin{array}{c}\text { Promedio mes } \\
\text { temperatura } \\
\text { máxima en }{ }^{\circ} \mathbf{C}\end{array}$ & $\begin{array}{c}\text { Promedio mes } \\
\text { temperatura } \\
\text { minina en }{ }^{\circ} \mathbf{C}\end{array}$ & $\begin{array}{c}\text { Promedio mes } \\
\text { humedad relativa } \\
\text { en } \%\end{array}$ \\
\hline Enero & 2 & 13,2 & 22,1 & 5,1 & 69,2 \\
\hline Febrero & 7 & 14,5 & 22,4 & 8 & 74,4 \\
Marzo & 5 & 14,7 & 21,5 & 8,9 & 74,7 \\
Abril & 3 & 14,6 & 21 & 10,6 & 83 \\
Mayo & 2 & 14,7 & 20,8 & 11 & 83,2 \\
Junio & 5 & 14 & 20 & 9,7 & 80,4 \\
Julio & 3 & 13,5 & 19,7 & 9,6 & 83 \\
Agosto & 2 & 13,5 & 19,3 & 9 & 79,8 \\
Septiembre & 2 & 13,4 & 19,3 & 9,4 & 81,6 \\
Octubre & 1 & 13,6 & 20,1 & 9,2 & 81,4 \\
Noviembre & 1 & 13,5 & 19,5 & 10 & 85,2 \\
Diciembre & 1 & 13,4 & 19 & 10,7 & 85,2 \\
\hline
\end{tabular}


morfológicas como fueron; para $R$. sanguineus adulto el color marrón claro, de $4 \mathrm{~mm}$, escudo sin ornamentación y abundantes puntuaciones, base del capítulo proyectada lateralmente, festones presentes y rodeados por una sutura marginal completa, coxa I con una hendidura formando dos espinas muy cercanas, coxas II - III con una pequeña protuberancia y coxa IV sin protuberancia (24,25,30); para R. (Boophilus) microplus por la presencia de espina caudal en el dorso, que puede observarse centralmente, la coxa I con dos espolones en forma de triangulo, el interno más ancho y largo que el externo, las coxas II y III presentan en su borde posterior una escotadura profunda, las placas adanales en el borde posterior con una escotadura de donde se origina una espina hacia el extremo interno, las placas accesorias son agudas en su borde posterior y dejan visible la espina caudal $(24,25)$; en el caso de Amblyomma maculatum, las características morfológicas para el espécimen adulto fueron: color marrón claro, de aproximadamente de 3,5 a $4 \mathrm{~mm}$, ornamentación del escudo representado por dos manchas laterales con punteado cerca de la base del capítulo, hipostoma 3/3, surco marginal completo delimitando los festones (30). Para el macho de esta especie adicionalmente se tuvo en cuenta: Coxa I con un gran espolón, coxa IV con un espolón pequeño, coxa II y III sin espolones, metatarsos del II al IV con dos espolones, trocánteres sin espinas y fórmula del hipostoma 3/3 (29), Figura 1.

\section{Discusión}

Este es el primer reporte de Rhipicephalus sanguineus, Rhipicephalus (Boophilus) microplus $\mathbf{y}$ Amblyomma maculatum Figura 1 dentro de la ciudad de Bogotá ectoparasitando a Canis familiaris y aunque se hallo 1 garrapata por canino, la tasa de infestación por estos artrópodos fue 4,25\%, una alta tasa por tratarse de animales de compañía, domiciliados, vinculados a planes de atención sanitaria, en una ciudad del trópico americano con altura de 2640 msnm, donde no se habían descrito antes. En Maracaibo, Venezuela, una ciudad del trópico costero americano, sólo con 5 meses de estudio, caninos con asistencia sanitaria tuvieron tasas infestantes por Rhipicephalus sanguineus de $9,8 \%$ con 2 a 55 garrapatas por animal, mostrando más infestados los canidos jóvenes y de razas puras (32); demostrando como su infestación varía ampliamente en caninos según la distribución biogeográfica, densidad y proporción, e individualmente incluso por raza, edad, y estilo de vida domiciliado o no (33).

Las garrapatas halladas están descritas dentro del territorio colombiano $R$. sanguineus y Rhipicephalus (Boophilus) microplus en Canis familiaris (26, 34) y $R$. sanguineus en Cerdocyon tous (35), pero no se encontraron reportes para Amblyomma maculatum, en la familia Canidae colombiana descritas para Canis familiaris en Costa Rica (36) y Venezuela (29).

Estudios de relación garrapata-hospedero canino en ciudades urbanizadas y capitales tan diversas como Varsovia - Polonia en Europa (37), MaputoMozambique en África (38) y Taipei - Taiwan en Asia (39) colectaron también R. sanguineus, confirmando la adaptabilidad del artrópodo a nuevos ambientes (33) que se relacionan llegadas a las ciudades inicialmente por movilización canina de regiones donde el artrópodo es prevalente $(37,38,40)$ hecho que no se descarta en Bogotá por la alta migración humana histórica al concentrar el $20 \%$ de población del país (41), ciudad con reconocida población canina callejera (42) de presencia inherentemente migrante hacia la población humana $(43,44)$.

Respecto a la detección de Rhipicephalus (Boophilus) microplus una garrapata asociada al ganado (45), estos animales y su transporte son los primeros distribuidores mecánicos de garrapatas hacia zonas libres como se ha demostrado en África (46). En en el caso de Bogotá es el ganado que llega entre otros de los llanos orientales, importante zona agropecuaria de Colombia, (47) con alta prevalencia bovina de Rhipicephalus (Boophilus) microplus (48); también se consideran aves migratorias como Bubulcus ibis, garza bueyera o ganadera (49) distribuidores mecánicos de este artrópodo (50). Estas aves han sido reportadas tanto en los humedales bogotanos $(8,51,52)$ como en llanos de Venezuela (53) y Colombia $(54,55)$.

Bubulcus Ibis quizá sea también vector de Amblyoma maculatum un ectoparásito de Hidrocehrus hidrochaeris, roedor también de distribución en llanos orientales (56). Con garrapatas en la ciudad, 
hembras y machos Tabla 2, más temperaturas elevadas de manera significativa en las últimas décadas (9) registros que distan de primeros reportes meteorológicos con máximas de $14^{\circ} \mathrm{C}$ y mínimas de $9.9^{\circ} \mathrm{C}$ tomados en 1807 (57) y una proporción canina-humana de 1:10.86 ligeramente por encima del promedio para la ciudad de Bogotá (42), junto a la biología de este artrópodo como ectoparásito de tipo generalista, con requerimiento de varios huéspedes para completar su ciclo (58) y capacidad de sobrevivir sin alimentarse por un año $(58,59)$, las condiciones están dadas para establecimiento de ciclos biológicos exitosos de garrapatas en la ciudad.

Nótese, en relación a la temperatura, la mayor colección de garrapatas se hizo entre enero y junio de 2010, con 24 especímenes, coincidiendo con promedios de temperatura mensuales de enero a junio más altos con $14,28{ }^{\circ} \mathrm{C}$ y con promedios máximos de $21.3^{\circ} \mathrm{C}$ para $R$. Boophilus microplus $14^{\circ} \mathrm{C}$ límite de temperatura sobre los cuales se reportan establecimiento de ciclos biológicos exitosos, Tabla 3 (60); para otras garrapatas hay demostraciones experimentales de mantenimientos de fases no parasíticas a temperaturas de $18^{\circ} \mathrm{C}$ con optimización similar a las establecidas en temperaturas más altas $(61,62,63,64)$ circunstancia que hace más factible la presencia de garrapatas en la ciudad; más aún, está establecido que la temperatura en Bogotá tiene cambios más extremos en la zona central urbanizada que las zonas periféricas menos urbanizadas y con mayor densidad de vegetación, lo cual determina estabilidad microclimática (9), estabilidad que ha sido reportada como necesaria para establecer ciclos reproductivos de garrapatas (59) y que justifica por las coordenadas geográficas de la clínica 3, la zona más periférica en el estudio y que se describe con la mayor vegetación de la localidad (8) coincidente con el mayor aporte de garrapatas (con 25 durante el año de estudio), Tabla 1.

Al momento de la consulta el estudio determinó como 12 de los 34 caninos con garrapatas, esto es $35,2 \%$, tuvieron signos de eritema migrans en el lugar de la picadura, acompańado de astenia, adinamia, depresión y fiebre, síntomas locales y sistémicos que si bien pueden relacionarse circunstancialmente con la presencia de la picadura de garrapatas, y los efectos hemolíticos de su saliva con capacidad de causar parálisis motora (65), también pueden relacionarse con infección presente y causada por Ehrlichia spp y Rickettsia spp (66).

Para Ehrlichia canis actualmente la especie de Ehrlichia spp más reportada en vertebrados en América del Sur (67) y demostrada en caninos de ciudades colombianas como Cali a $1000 \mathrm{msnm}$ con prevalencia de 49.5\% (68) y Medellín a 1500 msnm con una positividad de 1,58 \% (69); cerca a Bogotá, en Villeta, Cundinamarca a $842 \mathrm{msnm}$ y temperatura promedio de $26^{\circ} \mathrm{C}$, con coordenadas $5^{\circ} 053^{\prime \prime} \mathrm{N}, 74^{\circ} 28,29^{\prime \prime} \mathrm{W}$, hay prevalencia canina para las especie Ehrlichia chaffeensis en $31.8 \%$, y Rickettsia rickettsii en $18.2 \%$ (26), esta última agente etiológico de la fiebre manchada de las montanas rocosas, reportada en Estados Unidos, Argentina (70), Brasil, Canadá, Colombia (27, 28), Costa Rica, Estados Unidos y Panamá; para enfermedad principalmente relacionada con vectores como Dermacentor variabilis y $D$. andersonii (71) pero también hallada asociada a $R$. Sanguineus, vector en un brote de la enfermedad, en humanos y caninos de Arizona en el 2005; siendo los caninos quienes pudieron acercar la infección por Rickettsia spp. a asentamientos humanos habitados (72).

Para el año de 1999 en Alemania, se han hallado casos de ehrlichiosis canina e infestación por Rhipicephalus sanguineus en perros que nunca habían salido de Alemania (40) y para el año de 2003 en Francia se reporto la muerte de un habitante de la calle por fiebre de las montańas rocosas con hallazgos circunstanciales de ectoparasitismo por $R$ sanguineus, coincidiendo con temperaturas elevadas (más de 17 días con temperatura $\left.>35^{\circ} \mathrm{C}\right)(73,74)$. En este sentido, estudios de prevalencia sobre enfermedades transmitidas por garrapatas en caninos se hacen imperativos en la ciudad y el altiplano cundiboyacence, más cuando en la Sabana de Bogotá el calentamiento global ha incrementado la población y antrópicamente hay un cambio en el uso de suelo de la zona, por el tipo agropecuario que predispone la llegada de ganado $(75,76)$.

En Barquisimeto, Venezuela, ciudad a 614 msnm, con el verano de 1994 sucedió la primera infección por E. canis en humanos, demostrándose las garrapatas como vector de Ehrlichiosis por primera vez en Suramérica (77). En la primera década del 
siglo XXI con la más altas temperaturas que se tengan noticias en el planeta (78), cambios de los paradigmas que orientan las políticas en salud pública empiezan a ser necesarios; el riesgo poblacional de zoonosis trasmitidas por garrapatas no se conoce, y como demuestra esta investigación, incluso ya hay garrapatas (acari: ixodidae) de la especies Rhipicephalus sanguineus, Rhipicephalus (Boophilus) microplus y Amblyomma maculatum asociadas a caninos no migrantes en una ciudad históricamente una de clima frío.

\section{Agradecimientos}

Al fondo de Investigaciones de la Universidad Militar Nueva Granada y a los Médicos Veterinarios: Dr. Mauricio Rodríguez Dr. Jhon Freddy Rincón y Dra. Andrea Osorio.

\section{Referencias}

1. Nufio CR, McGuire CR, Bowers MD, Guralnick RP. Grasshopper Community Response to Climatic Change: Variation Along an Elevational Gradient. PLoS ONE. 2010; 5(9): 1-11

2. Bárcena A, Prado A, Samaniego J, Malchik S. Cambio climático: una perspectiva regional. Cumbre de la Unidad de América Latina y el Caribe (22 y 23 Febrero 2010: Riviera Maya, México), Santiago de Chile, Comisión Económica para América Latina y el Caribe (CEPAL). 2010. LC/L.3207

3. Classen AT, Norby RJ, Campany CE, Sides KE, Weltzin JF. . Climate Change Alters Seedling Emergence and Establishment in an Old-Field Ecosystem. PLoS ONE. 2010; 5(10):1-8

4. World Health Organization. Climate change and health. Fact sheet No. 266. Geneva: OMS 2010. [acceso 19 de diciembre de 2010]. Disponible en http://www.who.int/mediacentre/ factsheets/fs266/en/

5. Federal Government of Germany. German Strategy for Adaptation to Climate Change. Adopted by the German federal cabinet on 17th December 2008. [acceso 21 de diciembre de 2010]. En: http://www.bmu.de/files/english/pdf/application/ pdf/das_gesamt_en_bf.pdf

6. European Community EU action against climate Change, Leading global action to 2020 and beyond. 2009 [acceso $27 \mathrm{de}$ diciembre de 2010]. En: http://ec.europa.eu/clima/publications/ docs/post_2012_en.pdf

7. Githeko AK., Lindsay SW., Confalonieri UE, Patz JA. Climate change and vector-borne diseases: a regional analysis. Bull World Health Organ. 2000; 78(9):1136-1147

8. Parrado CC. Metodología para la ordenación del territorio bajo el prisma de sostenibilidad. Estudio de su aplicación en la ciudad de Bogotá D.C. [Tesis Doctoral]. Cataluńa: Departamento de Ingenieria, Mineria y Recursos Naturales, Universidad Politécnica de Cataluńa; 2001.

9. Ángel L, Ramírez A, Domínguez E. Isla de calor y cambios espacio-temporales de la temperatura en la ciudad de Bogotá. Rev. Acad. Colomb. Cienc. 2010; 34 (131):173-183.
10. Pabón J, Torres G. Impacto socioeconómico de los fenómenos El Niño y La Niña en la Sabana de Bogotá durante el siglo XX. Cuadernos de Geografía 2007; 16:81-94

11. Barcat JA. El calentamiento global, las garrapatas y la ehrlichiosis. Rev MEDICINA 2006; 66 (5): 489-491

12. Cortés J. Cambios en la distribución y abundancia de las garrapatas y su relación con el calentamiento global. Rev. Med. V et. Zoot. 2010; 57:65-75.

13. Horrocks L, Pye S, Searl A, Hunt A. Impacts of climate change in human health in Europe. PESETA-Human health study Paul Watkiss, European Commission Joint Research Centre Institute for Prospective Technological Studies EUR 24135. JRC Scientific and Technical Reports. 2009. [acceso 8 de enero de 2011]. En: http://ftp.jrc.es/EURdoc/JRC55393.pdf

14. Sánchez L, Mattar SV, González MT. Cambios climáticos y enfermedades infecciosas: nuevos retos epidemiológicos. Rev. MVZ Córdoba. 2009.14(3):1876-1885.

15. Gray JS, Dautel H, Estrada-Peña A, Kahl O, Lindgren E. Effects of climate change on ticks and tick-borne diseases in europe. Interdiscip Perspect Infect Dis. 2009; 1-12.

16. Olwoch JM, Van Jaarsveld AS, Scholtz CH, Horak IG. Climate change and the genus Rhipicephalus (Acari: Ixodidae) in Africa. Onderstepoort J Vet Res. 2007; 74(1):45-72.

17. Lindgren E, Tälleklint L, Polfeldt T. Impact of climatic change on the northern latitude limit and population density of the disease-transmitting European tick Ixodes ricinus. Environ Health Perspect. 2000; 108(2):119-23.

18. Gubler DJ. Resurgent vector-borne diseases as a global health problem. Emerg Infect Dis. 1998; 4(3):442-50.

19. Zavitsanou A, Babatsikou F, Koutis C. Crimean Congo Hemorrhagic fever: an emerging tick-borne disease. Health Science Journal. 2009; 3(1):10-18

20. Lukan M, Bullova E, Petko B. Climate warming and tick-borne encephalitis, Slovakia. Emerg Infect Dis. 2010; 16(3):524-526.

21. World Health Organization. Lindgren E, Jaenson TGT. Lyme borreliosis in Europe: influences of climate and climate change, epidemiology, ecology and adaptation measures., WHO Regional Office for Europe. 2006. 1-34.

22. Holden K, Boothby JT, Anand S, Massung RF. Detection of Borrelia burgdorferi, Ehrlichia chaffeensis, and Anaplasma phagocytophilum in ticks (Acari: Ixodidae) from a coastal region of California. J Med Entomol. 2003; 40(4):534-9.

23. Eskow ES, Krause PJ, Spielman A, Freeman K, Aslanzadeh J. Southern extension of the range of human babesiosis in the eastern United States. J Clin Microbiol. 1999; 37(6):2051-2052.

24. Benavides-Ortiz B, López-Valencia G. Clave pictórica para la identificación de garrapatas en Colombia y norte de Sudamérica. Corporación Colombiana de Investigación Agropecuaria. Centro de Investigación en Salud Animal CEISA. 2005. 1-16.

25. Osorno-Mesa E. Las garrapatas de la República de Colombia. Biomedica.2006; 26(3):317-336.

26. Hidalgo M, Vesga JF, Lizarazo D, Valbuena G. Short Report: A Survey of Antibodies against Rickettsia rickettsii and Ehrlichia chafeensis in Domestic Animals from a Rural Area of Colombia. Am J Trop Med Hyg. 2009; 80(6):1029-1030.

27. Patino L, Afanador A, Paul JH. A spotted fever in Tobia, Colombia. Am J Trop Med. 1937; 17:639-53.

28. Hidalgo M, Orejuela L, Fuya P, Carrillo P, Hernandez J, Parra E, Keng C, Small M, Olano JP, Bouyer D, Castaneda E, Walker D, Valbuena G. Rocky Mountain spotted fever, Colombia. Emerg Infect Dis. 2007; 13(7):1058-60. 
29. Manzanilla JE, García M, Moissant EA, García F, Tortolero E. Dos especies de garrapatas del género Amblyomma (Acari: Ixodidae) en perros del estado Aragua, Venezuela. Entomotropica. 2002; 17(2):177-180.

30. Clavijo JJ, Vásquez CL, Coromoto-Valera N. Garrapatas duras de los géneros Amblyomma Koch y Rhipicephalus Koch (Acari: Ixodidae) presentes en la Colección de Zoología Agrícola, Decanato de Agronomía, Universidad Centro Occidental Lisandro Alvarado Venezuela. Entomotropica. 2009; 24(1):41-50.

31. Tutiempo Network S.L. Clima en Bogota/ el dorado durante el año 2010. [online] 2011. [Consultado enero 2 de 2011]; Disponible en: http://www.tutiempo.net/clima/Bogota_Eldorado/2010/802220.htm.

32. Ramirez-Barrios RA, Chacin E, Barboza G, Fernandez G, Valera Z, Villalobos A, Angulo-Cubillan F. Garrapatas (Acari: Ixodidae) Recolectadas de Caninos Bajo Asistencia Veterinaria en Maracaibo, Venezuela. Rev Cient (Maracaibo). 2008; 18(3):267:270.

33. Dantas-Torres F. Biology and ecology of the brown dog tick, Rhipicephalus sanguineus. Parasites \& Vectors. 2010; 3:26.

34. Paternina LE, Diaz-Olmos Y, Paternina-Gmoez M, Bejarano EE. Canis familiaris, un nuevo hospedero de Ornithodoros (A.) puertoricensis fox, 1947 (ACARI: IXODIDA) en Colombia. Acta Biol Colomb. 2009; 14(1):153-160.

35. Torres-Mejía AM, de la Fuente J. Risks associated with ectoparasites of wild mammals in the department of Quindío, Colombia. Intern J Appl Res Vet Med. 2006; (4)3:187-191.

36. Álvarez-Calderón A, Hernández-Fonseca V, Hernández-Gamboa J. Catálogo de garrapatas suaves (Acari: Argasidae) y duras (Acari:Ixodidae) de Costa Rica. Brenesia. 2005; 63 (64):81-88.

37. Zygner W, Wedrychowicz H.Occurrence of hard ticks in dogs from Warsaw area. Ann Agric Environ Med. 2006; 13(2):355-9.

38. Neves L, Afonso S, Horak IG.Ixodid ticks on dogs in southern Mozambique. Onderstepoort J Vet Res. 2004; 71(4):279-83.

39. Wu TJ, Sun HJ, Wu YC, Huang HP. Prevalence and Risk Factors of Canine Ticks and Tick-Borne Diseases in Taipei, Taiwan. JVCS. 2009; 2(3):75-78.

40. Gothe, R. Rhipicephalus sanguineus (Ixodidae): frequency of infestation and ehrlichial infections transmitted by this tick in dogs in Germany; an epidemiological study and consideration. Wiener Tierarztliche Monatsschrift. 1999; 86(2):49-5.

41. Utria RD. Metropolización de la sabana de Bogotá. Martes del planetario, Sociedad Geográfica de Colombia. Bogota; 1998; 1: $1-25$.

42. Codeisa. Comportamiento de la población canina del distrito capital analizando tasas de fecundidad, natalidad y mortalidad y la relación hombre-animal. Secretaria Distrital de Salud Santa Fe de Bogotá. 1999; 1: 1-14.

43. Valadez-Azua R, Mendoza-España V. El perro como legado cultural. Nuevos aportes. 2005; 2:15-35.

44. Girard-Rheault M. Zooarqueologia de los perros (Canis lupus familiaris L.) en Canada. Archaebios. 2009; 3(1):46-54.

45. Food and Agriculture Organization. Nari A. Estado actual de la resistencia de Boophilus microplus en America latina y el Caribe. Reunión anual del Grupo de Trabajo sobre Resistencia Parasitaria. México. 2005; 1.15.

46. Madder M, Thys E, Achi L, Touré A, De Deken R. Rhipicephalus (Boophilus) microplus: a most successful invasive tick species in West-Africa. Exp Appl Acarol. 2011; 53(2):139-45.

47. Ministerio de agricultura y desarrollo rural. Republica de Colombia. Uribe C, Ramírez A. Evaluación de medio término a subproyectos cofinanciados en las convocatorias nacionales para la cofinanciación de proyectos de Investigación, desarrollo tecnológico e innovación para el sector agropecuario por cadenas productivas. 2010. Inf Tec 1: 1-143.

48. Villar CE. Aspectos básicos para el manejo integral del parasitismo en bovinos. Corporación colombiana de investigación agropecuaria, CORPOICA. 1998. (1) 1-8.

49. Gómez de Silva H, Oliveras A. Medellín RA. Bubulcus ibis ibis. Vertebrados superiores exóticos en México: diversidad, distribución y efectos potenciales. (Tesis). Instituto de Ecología, Universidad Nacional Autónoma de México. 2005.

50. Briceńo CL. La garza del ganado (Bubulcus ibis) como posible depredador y vector mecánico de la garrapata bovina Boophilus spp. (Tesis). Instituto Tecnológico de Costa Rica. Departamento de Agronomía. 1990

51. Sociedad Geográfica de Colombia. Preciado AP. La estructura ecológica principal de la Sabana de Bogotá. Martes del planetario, Bogota; 2000. Inf Tec 1: 1-37.

52. Instituto de investigación de recursos biologicos Alexander Von Humbolt. Andrade ME. Benitez-Castañeda H. Los humedales de la sabana de Bogotá: area importante para la conservación de las aves de Colombia y el mundo. 2005. Inf Tec 1: 1-38.

53. Frederick P, Bildstein K Foraging ecology of seven species of Neotropical ibises (Threskiornithidae) during the dry season in the llanos of Venezuela. Wilson Bull. 1992; 104: 1-21.

54. Hilty SL, Brown WL. A guide to the birds of Colombia. Princeton University Press, Princeton, EUA. 1986.

55. Instituto de Investigación de Recursos Biológicos Alexander von Humboldt. Romero MH, Maldonado-Ocampo JA, Bogotá-Gregory JD, Usma JS, Umaña-Villaveces AM, Murillo JI, RestrepoCalle S, Álvarez M, Palacios-Lozano MT, Valbuena MS, Mejía SL, Aldana-Domínguez J, Payán E. Informe sobre el estado de la biodiversidad en Colombia 2007-2008: piedemonte orinoquense, sabanas y bosques asociados al norte del río Guaviare. Bogotá, Colombia. 2009.

56. Latorre SO, Mesa LE, Villar-Cleves C. Parásitos gastrointestinales en chigüiro (Hydrochaeris hydrochaeris). Acovez. 2007; 37(1):1-7.

57. Pabón-Caicedo JD. El clima de Colombia durante los siglos xvixix a partir de material histórico. Parte I: Inventario de fuentes de información. Cuadernos de Geografía. 2006; 15:75-92.

58. Strong A. Tick Host-Seeking Behavior. Insect Behavior. 2005; 1: $1-15$.

59. Anderson JF, Magnarelli LA. Biology of Ticks. Infect Dis Clin N Am. 2008; 22:195-215.

60. Estrada-Peńa A, García Z, Fragoso Sánchez H. The distribution and ecological preferences of Boophilus microplus (Acari: Ixodidae) in Mexico. Exp Appl Acarol. 2006; 38: 307-316.

61. Chacon SC, Correia PG, Barbieri FS, Daemon E, Faccini JL. Effects of three constants temperatures on the free living phase of Amblyomma cajennense (Fabricius, 1787) (Acari: Ixodidae). Rev Bras Parasitol Vet. 2003; 12(1): 13-20.

62. Bellato, V. Daemon E. Efeitos de três temperaturas sobre a fase não parasitária de Rhipicephalus sanguineus (Latreille, 1806) (Acari: Ixodidae). Rev Bras Parasitol Vet. 1997; 6(1):21-27.

63. Bastos KM, Daemon E, Faccini JL, Cunha DW. Efeito de diferentes temperaturas sobre a fase não parasitária de Dermacentor (Anocentor) nitens (Neumann, 1897) (Acari: Ixodidae) em condiçôes de laboratorio.. Rev Bras Parasitol Vet. 1996; 5(1):29-32.

64. De La Vega R, Diaz G, Da Fonseca AH. A multivariate analysis of Boophilus microplus (acari: ixodidae): non-parasitic phase. Rev Salud Anim. 2010; 32(2):89-96. 
65. Mans BJ, Gothe R, Neitz AW.Biochemical perspectives on paralysis and other forms of toxicoses caused by ticks. Parasitology. 2004; 129 Suppl: S95-111.

66. Fritz CL. Emerging tick-borne diseases. Vet Clin North Am Small Anim Pract. 2009; 39(2):265-78.

67. Spolidorio MG, Labruna MB, Machado RZ, Moraes-Filho J, Zago AM, Donatele DM, Pinheiro SR, Silveira I, Caliari KM, Yoshinari NH. Survey for tick-borne zoonoses in the state of Espirito Santo, southeastern Brazil. Am J Trop Med Hyg. 2010; 83(1):201-206.

68. Silva R, Sanchez N, Loaiza A. Reporte de presentación de Erlichia canis en muestras sanguíneas de caninos en la ciudad de Cali, Colombia. Veterinaria y Zootecnia. 2008; 2(1):39-43.

69. González MS, Caraballo AJ, Arango J. Frecuencia de Ehrlichia canis y su relación con los parámetros serológicos y hematológicos en caninos en Medellín (Colombia) Rev Colomb Cienc Pecu. 2009; 22(3):558-559.

70. Parola P, Paddock CD, Raoult D. Tick-borne rickettsioses around the world: emerging diseases challenging old concepts. Clin Microbiol Rev. 2005; 18(4):719-56.

71. Dantas-Torres F. Rocky Mountain spotted fever. Lancet Infect Dis. 2007; 7(11):724-32.

72. Demma LJ, Traeger MS, Nicholson WL, Paddock CD, Blau DM, Eremeeva ME, Dasch GA, Levin ML, Singleton J Jr, Zaki
SR, Cheek JE, Swerdlow DL, McQuiston JH. Rocky Mountain spotted fever from an unexpected tick vector in Arizona. N Engl J Med. 2005; 353(6):587-94.

73. Rovery C, Brouqui P, Raoult D. Questions on Mediterranean spotted fever a century after its discovery. Emerg Infect Dis. 2008; 14(9):1360-7.

74. Hemmersbach-Miller M, Parola P, Raoult D, Brouqui P. A homeless man with maculopapular rash who died in Marseille, France. Clin Infect Dis. 2004; 38(10):1412-1493.

75. Cortés S, Van der Hammen T, Rangel O. Comunidades vegetales y patrones de degradación y sucesión en la vegetación de los cerros occidentales de Chía-Cundinamarca-Colombia. Rev Acad Colomb Cienc. 1999; 89(23):529-554.

76. Instituto de investigación de recursos biológicos Alexander von Humboldt. Rodríguez-Eraso N, Pabón-Caicedo JD, BernalSuárez NR, Martínez-Collantes J. Cambio climático y su relación con el uso del suelo en los Andes colombianos 2010. InfTec 1-84.

77. Perez M, Rikihisa Y, Wen B. Ehrlichia canis-like agent isolated from a man in Venezuela: antigenic and genetic characterization. J Clin Microbiol. 1996; 34(9):2133-9.

78. National Aeronautics and Space Administration. NASA. United States. Research News 2009: Second Warmest Year on Record; End of Warmest Decade. [Consultado Dic 26 de 2010]; Disponible en: http://www.giss.nasa.gov/research/news/20100121/
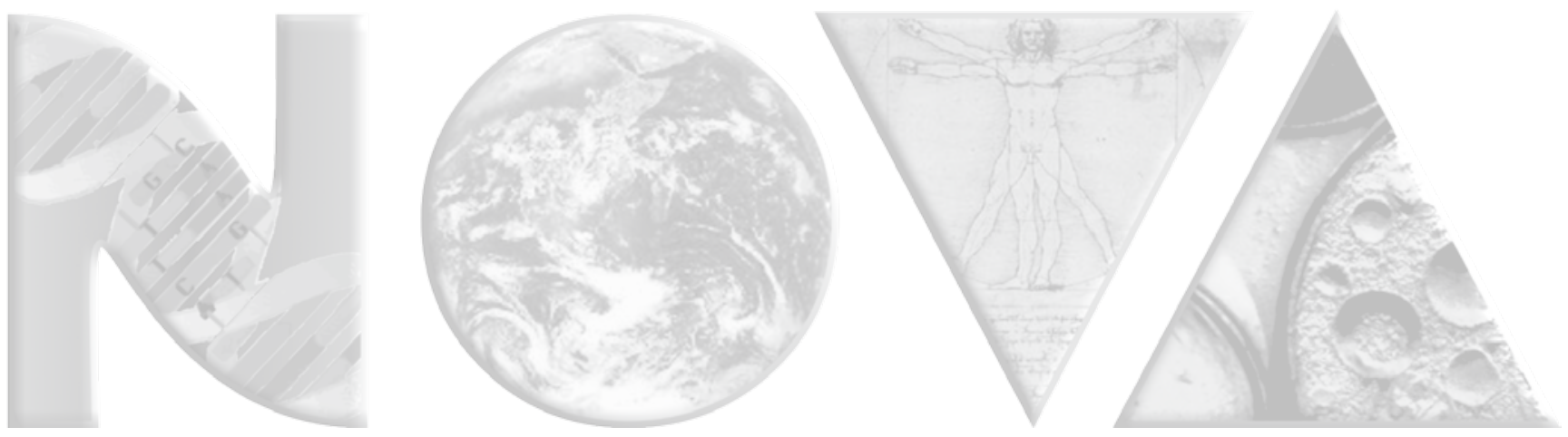